\title{
ANÁLISIS DE LA ESCUELA RURAL ESPAÑOLA: ESTUDIO DE CASO
}

\author{
Marta Recio GIL ${ }^{1}$ \\ Teresa Sacristán ALFARO² \\ María Odete de MATTOS ${ }^{3}$ \\ Eladio Sebastián HEREDERO ${ }^{4}$
}

RESUMEN: En este trabajo de investigación pretendemos estudiar los elementos que envuelven a la escuela rural, desde su concepto y cómo es su organización hasta como trabaja el equipo directivo, los profesores itinerantes o cómo se trabaja con la diversidad de alumnos que hay en una misma aula. Esta investigación ofrece además una descripción de la situación de las escuelas rurales; trabajo logrado a través de la observación de una escuela y la recopilación de datos sobre la realidad del maestro, del alumno, de la normativa y de la estructura física de esta escuela rural. Para ello, nos hemos acercado a una escuela rural, situada en un pueblo de Guadalajara, Albalate de Zorita, denominado Colegio Rural Agrupado "La Colmena" cabecera de tres colegios de la provincia situados en Albalate de Zorita, Almonacid de Zorita e Illana, situada en Castilla La Mancha una región que ha avanzado bastante en la línea de dar respuesta a este tipo de escuelas.

PALABRAS CLAVE: Organización escolar. Colegio Rural Agrupado (CRA). Centro de Recursos y Asesoramiento a la Escuela Rural (CRAER). Centros Rurales de Innovación Educativa (CRIE). Escuelas unitarias/aulas unitarias. Profesores itinerantes.

\section{Introducción}

Por escuela rural no debemos entender simplemente la escuela de los pueblos, de las localidades más pequeñas, sino la respuesta educativa y organizativa a un constante cambio en sus necesidades y características singulares, acorde con los cambios en la sociedad y de las personas que viven en ellos.

Sin embargo esta escuela ha quedado un tanto marginada a lo largo de la historia, sobre todo en materia de calidad de su educación, ya que debería merecer el mismo tratamiento social y económico que las escuelas urbanas. Enseñar en la escuela rural es algo complejo y diferente a enseñar en las escuelas de las ciudades, y más cuando se trabaja en aulas en las que hay pocos alumnos y normalmente con diferentes niveles dentro de una misma aula.

Los aspectos organizativos son otra de las claves de la forma singular de trabajar en la escuela rural, desde los que hacen referencia a la estructuración de los centros y aulas como

\footnotetext{
1 Alumna de $3^{\circ}$ de Magisterio. Universidad de Alcalá. Alcalá de Henares - Madrid - España. 28801 teresa.sacristan@alu.uah.es

${ }^{2}$ Alumna de $3^{\circ}$ de Magisterio. Universidad de Alcalá. Alcalá de Henares - Madrid - España. 28801.

${ }^{3}$ Profesora brasileña visitante del Departamento de Didáctica de la Universidad de Alcalá en el curso 2009.

4 Universidad de Alcalá. Departamento de Didáctica. Alcalá de Henares - Madrid- España. 28801. eladio.sebastian@uah.es
} 
los referidos a los aspectos de recursos humanos y materiales, sin olvidar los relativos a sus órganos de participación y dirección o los propiamente administrativos. Por otro lado, el docente constituye una pieza clave para interpretar la realidad escolar actual y modificar los enfoques tradicionales que envuelven esta escuela.

A pesar de ello, hoy en día los relatos y posiciones de los participantes que han hecho posible esta investigación son de queja, con falta de apoyos y de respuesta personalizada, como ya escribiera Pliya (1990) “[...] salvo casos excepcionales, los niños de las poblaciones rurales carecen del acompañamiento material, financiero y cultural de la escolaridad, lo que atenta contra la calidad educativa de las escuelas rurales [...]”, al igual que coinciden en los aspectos positivos. Feu (2003) insiste en acentuar las virtudes de la escuela y el medio rurales,

(la cercanía con todos los miembros de la comunidad educativa), así como otros que se expondrán a continuación.

Por ello, hacemos nuestras aquellas palabras de Boix Tomás (1995, p.13), quien ya señalo claramente que la educación rural necesita un cambio en sus estructuras básicas: “conceptualización, finalidades, actuaciones” hecho que ha avanzado considerablemente pero que todavía necesita bastantes más, por lo que se hace necesario seguir poniendo en marcha procesos innovadores que se adapten a las múltiples realidades socioculturales, lo que supone también considerar diferentes tipos de escuelas y de contextos.

Nos hemos querido centrar en un centro rural emplazado en la Comunidad de Castilla La Mancha por ser esta una región que por sus características está trabajando en ofrecer una respuesta y organización personalizada para la escuela rural, a pesar de que todavía hay margen para el crecimiento e innovación.

\section{Estado de la Cuestión}

Vamos a empezar por estudiar en primer lugar las características de estos centros rurales denominados Colegios Rurales Agrupados (CRA) y también los centros que existen para su apoyo los Centro Rural de Innovación Educativa (CRIE) o los más novedosos y singulares Centros de Recursos y Asesoramiento a la Escuela Rural (CRAE) que aunque se trata de una realidad exclusiva de la Comunidad Autónoma de Castilla La Mancha tiene un paralelismo con otras instituciones similares, Centros de Profesores, en el resto de la geografía española.

Pero la singularidad de la escuela rural esta constituida por todas aquellas escuelas, que todavía siguen estando en muchas localidades de nuestra geografía que de forma aislada e 
individual atienden a la población rural de una determinada zona. Estas Escuelas ya sean completas o incompletas representan y han representado la característica dominante de España hasta la llegada de la industrialización indiscriminada y los movimientos migratorios a las grandes ciudades de los años 60 a 80 del siglo pasado.

Estas escuelas, alejadas de las grandes urbes y del acceso a innúmeros recursos, eran llevadas en muchos casos por profesores que dedicaban su tiempo y alma a la educación de sus pupilos y que suplían la falta de recursos mediante ideas imaginativas y sobre todo su tiempo. Pero desde hace algunos años esta concepción cambió y se empezaron a dar cuenta de que esta singularidad española no podía quedar ajena a los avances ni a los recursos de las otras escuelas, todo ello bajo la filosofía de una educación igual para todos y sobre todo de reconocimiento de la singularidad de esta escuela frente a movimientos que llevarían a una desertización de las zonas rurales.

Es al inicio de los años setenta cuando algunas iniciativas toman cuerpo, en primer lugar para dar mas acceso se crean los Escuelas Hogar, con el fin de proporcionar recursos a los niños para que puedan seguir sus estudios. Aunque la separación de los niños de sus padres y entorno cinco días a la semana no se evaluó como la mejor solución.

Al mismo tiempo se construyeron más institutos en zonas rurales en los que pudiesen continuar con calidad sus estudios. Este cambio en la concepción de la enseñanza supuso la dotación a los centros que escolarizaban a estos alumnos de más recursos de profesores y de medios, y sobre todo el reconocimiento de su singularidad.

Ya en los años 80 con la creación de algunos servicios paralelos a la escuela como los Centros de Profesores, los Servicios de Orientación Educativa y Psicopedagógica; los Centros de Recursos a la escuela rural o los propios Colegios Rurales Agrupados esta escuela rural recibió su gran impulso. Pero no por ello podemos decir que el camino está ya finalizado, de ahí esta investigación.

Desde hace años los titulares de los Ministerios de Educación han querido mantener la idiosincrasia propia de nuestros pueblos y zonas rurales, de ahí que se hayan acuñado frases que constituyen toda una declaración de intenciones y la formulación de una orientación específica para la definición de las correspondientes políticas educativas, como esta que se recoge en la historia de estas instituciones "Es preciso conciliar la calidad educativa con la calidad de vida", lo que supone el reconocimiento del derecho de las zonas rurales a una educación de calidad, compatible con las características de su hábitat y de sus modos de vida y, a un tiempo, el refuerzo de la importancia del servicio educativo como elemento de 
ordenación del territorio en un momento en el que el riesgo de despoblamiento total constituye un hecho cierto.

Programas como el de Educación Compensatoria o los Programas de Integración promovidos por el MEC desde la década de los 80 también sirvieron para apoyar y ofrecer recursos a esta escuela rural y paliar de alguna forma las desigualdades que sufrían. La creación de profesores itinerantes, aunque el modelo tiene todavía posibilidades de mejora, han sido otro aliciente importante para su desarrollo y mejora de la calidad.

En la actualidad más reciente las Consejerías de Educación de las Comunidades Autónomas donde existen estas realidades están haciendo de esto políticas propias, singulares y modos de enfrentarse a una realidad a la que no pueden, ni desean, ser ajenos. En la Comunidad Autónoma estudiada, Castilla La Mancha, en la que gran parte del territorio es rural aunque no es grande la población que vive en estas zonas rurales, esto constituye un programa propio dentro su modelo educativo con organizaciones y recursos singulares y adaptados como veremos más adelante.

\section{Colegio Rural Agrupado (CRA)}

Un Colegio Rural Agrupado (CRA) es un centro educativo de carácter singular, ya que está constituido por varias escuelas incompletas situadas en varias localidades diferentes y donde se imparten las enseñanzas de Educación Infantil y Educación Primaria en el entorno rural español.

El concepto de Colegio Rural Agrupado nace en los años 80, la evolución de esta modalidad de escolarización, específica de las zonas rurales, provocó la aceptación de este modelo organizativo frente a otros que suponían el traslado de alumnos de zonas rurales a zonas urbanas aunque solo fuera por periodos cortos de tiempo. Por entender su trascendencia, entre el curso 1990/91 y el 1997/98 el número de CRA en el territorio de gestión directa del Ministerio de Educación y Cultura se multiplicó por 9 y, en la actualidad, 66.099 alumnos reciben sus enseñanzas primarias en estos centros, lo que representa el 7 \% del total del alumnado correspondiente a dichos niveles educativos. Todo ello a pesar del efecto de caída demográfica, que es particularmente intenso en bastantes zonas de este medio. Por otra parte, 7.321 maestros están adscritos al sistema de CRA, lo que supone el 10,7\% del total del profesorado de educación infantil y primaria. Hay Comunidades Autónomas que han desarrollado este modelo al máximo de su exponente y otras que siguen ofreciendo el modelo 
de colegios incompletos con apoyos en las áreas de especialistas desde centros de recursos o desde Colegios completos.

En muchos de estos pueblos con poca población no hay alumnos suficientes como para mantener una infraestructura educativa tradicional, por lo que se establecen colegios rurales de pocas aulas (normalmente entre 1 y 3 ) donde los alumnos dan varios cursos simultáneamente, y una relación entre los distintos centros de una misma zona para compartir convergencias. Normalmente esta organización tiene un centro cabecera, que está en la localidad más importante de la comarca, que asume las tareas de gestión y administración del CRA y donde suele impartir docencia el director.

Los CRA, al igual que cualquier otro colegio tienen equipo directivo (Director/a, Jefe(a estudios y secretario/a), profesores generalistas y especialistas, tutores y todo el personal de administración y servicios necesario, las plantillas se crean en función de las variables aulas, centros o escuelas y distancia entre las mismas. En cada aula de los CRA se suelen integrar dentro de la misma clase a varios cursos, impartidos todos ellos por el mismo profesor, el que sean centros incompletos es una característica de estos. Cada colegio puede tener una o más aulas, si tiene solamente un aula se denomina centro unitario.

Normalmente todos los colegios o aulas que integran el CRA suelen tener recursos materiales propios como biblioteca, audiovisuales, ordenadores, material de educación física, etc y las dotaciones de cada uno de ellos depende la estructura original del colegio, algunos disponen de varias aulas, despachos, pistas deportivas, patios de recreo,.. es frecuente que además algunos usen infraestructuras municipales de forma compartida en horarios escolares, en otros casos las instalaciones son mínimas, con apenas una o dos aulas y servicios como toda la dotación de la que disponen.

Además del equipo directivo completo, en estos centros hay dos tipos de puestos: los ordinarios, que son ocupados por profesores estables en las correspondientes localidades y desempeñan la función de tutores de los grupos de alumnos, y los itinerantes, propios de profesores especialistas de Idioma extranjero, Educación Física y Música, y en su caso de apoyo a la Educación Infantil, Pedagogía Terapéutica o Audición y Lenguaje que imparten su especialidad en varias localidades del CRA. Que el profesorado ha recibido una formación inicial y permanente deficiente para trabajar en escuelas rurales no parece ninguna novedad. Es difícil encontrar un texto que describa a su profesorado y que no se dirija a las facultades y escuelas universitarias como culpables del desencuentro entre formación y realidad profesional, y este estudio insiste de nuevo en ello. El trabajo en aulas con multigraduación (BUSTOS JIMÉNEZ, 2006) responde a una peculiaridad de este tipo de escuelas que no se 
da en otros contextos escolares y, por lo tanto, requiere una base formativa específica. No es casual que en el último Informe sobre el Estado y Situación del Sistema Educativo en España (2007) se inste a las administraciones educativas a que atiendan de manera adecuada a los maestros y las maestras, insistiendo en la formación permanente para prestar el servicio educativo en el sector rural.

Cada CRA suele pertenecer a la misma demarcación atendía por el Servicio de Inspección Educativa, por los Equipos de Orientación u Orientador en el caso de Comunidades Autónomas que así lo tienen organizado y por el Centro de Profesores o CRAER en el caso de Castilla La Mancha.

\section{Centros Rurales de Innovación Educativa (CRIE)}

Nacieron en el curso 1983-84, acogidos al Real Decreto de Educación Compensatoria, de 13 de abril de 1983, que el M.E.C. puso en marcha para corregir y compensar situaciones de desigualdad educativa en que se encontraban algunos sectores de población debido a razones socioeconómicas, de procedencia social, de pertenencia a grupos de minorías étnicas o de ubicación en áreas rurales desfavorecidas. Fueron creados y regulados legalmente por la Orden Ministerial de 29 de abril de 1996. (BOE 11.05.96), actualmente se regulan específicamente por cada Comunidad Autónoma (CCAA) y no todas las CCAA los han desarrollado.

En los CRIE se desarrollan actividades de carácter curricular que por su naturaleza son más difíciles de cursar en los Centros Rurales Agrupados (Tecnología, Música, Informática, Laboratorio de Ciencias, etc.), así como actividades complementarias (charlas, animación a la lectura, dinámicas de grupos, etc.) según señala ROCHE (1992) en su trabajo sobre Los Centros Rurales de Innovación Educativa de Teruel (CRIET): un intento de solución al problema de la Escuela Rural.

Dichos centros complementan y refuerzan el papel de los Colegios Rurales Agrupados de una misma provincia. Su plantilla docente está compuesta por una dotación de entre 3 y 6 maestros y su finalidad es contribuir a la mejora de la socialización de los alumnos de las zonas rurales y realizar actividades que desarrollen y complementen la acción educativa que se lleva a cabo en los CRA.

Cada convivencia está formada por grupos de 50 alumnos del mismo nivel educativo procedentes de distintas zonas y escuelas rurales, para ello, se establece un calendario de convivencias periódicas de alumnos, que son transportados a los CRIE desde sus localidades y durante las cuales comparten juegos y experiencias, ofreciendo al alumnado la posibilidad 
de convivir con otros niños de su edad, favoreciendo su desarrollo personal, compensar las carencias, aportar recursos o potenciar aspectos que dichos alumnos debido a su procedencia tenían dificultad en alcanzar.

De forma general, los alumnos que asisten al CRIE provienen de zonas rurales, pero en los últimos años, los cambios demográficos y sociales han provocado que la procedencia de los niños que asisten al CRIE empieza a ser de origen urbano, lo que da lugar a planteamientos diferentes.

\section{Centros de Recursos y Asesoramiento a la Escuela Rural(CRAER)}

Los Centros de Recursos y Asesoramiento a la Escuela Rural (CRAER) son unos centros de formación del profesorado y de apoyo a la escuela rural específicos de la Comunidad Autónoma de Castilla La Mancha ${ }^{5}$, en otras comunidades los mismos Centros de Profesores (o su denominación específica) hacen este apoyo, aunque por su singularidad los hemos traído a esta investigación.

La organización y funcionamiento de estos centros, está regulada por la orden del 8 de marzo de 2006, de la Consejería de Educación y Ciencia de Castilla La Mancha. Este tipo de centros fue creado para garantizar la fluidez de las relaciones y el desarrollo de los centros educativos de las zonas rurales, y para ello, era necesario asegurar que todos los centros tuviesen una referencia que facilitase su relación con el CRAER y sirviese para encauzar sus demandas, además de garantizar unos servicios, como es el de orientación de una forma eficaz y coordinada.

Los CRAER, anteriormente eran Centros de Profesores y Recurso pero debido a la realidad de Castilla La Mancha y al objeto de atender a estas zonas rurales, dispersas de población y alejadas de las grandes urbes, se han configurado de forma que puedan atender a estos centros tanto en su parte de formación continua como de asesoramiento y de facilitación de recursos para su desarrollo e impulso. La dotación de base se compone de dirección y asesorías (Lingüística, Científica y de Tecnologías Educativas) pero cuentan con la singularidad de una asesoría de orientación compuesta por especialistas de Psicología y Pedagogía y Profesorado de Servicios a la Comunidad que atienden directamente a los centros en sus necesidades de orientación, al haber desaparecido los equipos de orientación en esta

\footnotetext{
${ }^{5}$ Estos centros solo se encuentran en Castilla La Mancha, con un total de 10 centros repartidos por toda la comunidad. Se encuentran 2 en Guadalajara, 1 en Cuenca, 2 en Albacete, 3 en Ciudad Real y 2 en Toledo.
} 
Comunidad Autónoma y no tener alumnado suficiente para ser dotados de un orientador a tiempo completo.

Los asesores de orientación de los CRAER, a través de su presencia semanal en los centros docentes, asumirán la referencia de los mismos. La intervención del resto de asesores estará asociada al desarrollo de programas específicos. La intensidad de la presencia del asesor en un centro determinado, es el criterio básico para la atribución de la referencia.

\section{Objetivos de la Investigación}

Llevando a cabo esta investigación pretendemos conseguir los siguientes objetivos:

- Conocer el funcionamiento de un CRA.

- Conocer las diferencias de un CRA y un colegio urbano en cuanto a organización, personal, normativa.

- Conocer las singularidades y contenidos que sean diferentes del PEC de un CRA y el PEC de un colegio urbano.

- Conocer como se elabora y qué recoge la PGA de un CRA.

- Conocer como se atiende a las características en la Programación Didáctica de tantos alumnos en una misma clase.

\section{Instrumentos}

Para realizar esta investigación acudimos presencialmente al Colegio Santa Cruz, perteneciente al CRA La Colmena. Allí obtuvimos información gracias a los diferentes profesores que allí trabajan, así como de la Directora y Secretaria del colegio, que nos facilitó diversos datos y documentos para llevar a cabo nuestra investigación a través de la entrevista y de una mesa de discusión.

Para complementar esta investigación se desarrollaron además otras dos acciones, de un lado un análisis documental de los diferentes instrumentos organizativos del centro; PEC, PGA, Programaciones Didácticas... Y una observación directa del trabajo e instalaciones del CRA La Colmena.

\section{Análisis de los resultados}

Empezaremos haciendo un poco situación del contexto de la investigación. El CRA “La Colmena”, actualmente está integrado por tres centros, el Colegio de Illana (a $21 \mathrm{Km}$ de la cabecera), el Colegio de Almonacid de Zorita (a unos 6Km de la cabecera) y el Colegio 
“Santa Cruz”, en el propio Albalate, y que es la cabecera del CRA, elegida como tal por su ubicación más central. Va a ser el sitio de referencia donde está el director, donde se reúne el claustro y Consejo Escolar y donde se guarda la documentación del centro, etc. Se eligió sobre todo su buena situación, que está más próxima al resto de centros del CRA y además que era el colegio más amplio, debido a que dispondrá de la centralización de recursos. En un principio, el Colegio Santa Cruz era un colegio completo, de concentración con transporte escolar y comedor al que acudían alumnos de las zonas más dispersas (localidades y fincas) de Zorita y Tarancón. El colegio de Illana, siempre fue un colegio incompleto que en principio estuvo unido al CRA de Almoguera, pero que una nueva organización lo asignó a este nuevo CRA. El colegio de Almonacid fue un centro independiente durante bastantes años debido al gran número de alumnos y de población facilitada por el trabajo de la Central Nuclear. Mas tarde, debido al cierre de la Central Nuclear, el número de niños matriculados descendió notablemente por lo que se unió al CRA de Pastrana y posteriormente al nuevo CRA La Colmena, quedando así los colegios de Illana, Almonacid y Albalate agrupados en este desde el año 2001.

En la actualidad, curso 08/09 hay un total de trece aulas repartidas de la siguiente forma Albalate seis, Almonacid cinco, Illana dos, con un total de 195 alumnos, repartidos 90 en Albalate, 75 en Almonacid y 30 en Illana.

Este curso escolar, el centro dispone de 24 profesores, trece son tutores, ocho de ellos itinerantes y el equipo directivo. Los itinerantes son:

- Profesor de Audición y Lenguaje

- Profesor de Pedagogía Terapéutica

- Orientador

- Profesor de inglés

- Profesor de religión

- Profesor de música

- Profesor de Educación Física

- Profesor de apoyo a Infantil.

A todos ellos, debido a la necesidad de desplazarse (itinerancia) para atender la educación en todas las localidades y dependiendo de la distancia que recorran se les otorgan horas libres en compensación. Todos tienen un total de 29 horas semanales de las que 23 horas lectivas y 6 complementarias, (4 para las exclusivas -coordinación ciclo, formación, 
claustro, CCP y reunión con padres- y 2 para la preparación de material). La queja más común por parte de estos itinerantes es que la amplitud de las horas no lectivas no llega a ser cumplida en su totalidad, ya que es muy usual que tengan que destinarlas a hacer sustituciones y apoyos.

Se puede observar que la plantilla y aulas en este CRA, es una característica de estas agrupaciones, es cambiante dependiendo del número de alumnos de los que se dispongan. Este hecho es especialmente visible en el caso de alumnos de Illana, donde los alumnos suelen variar por la cercanía a una urbanización y cuya población es muy inestable. En otros casos ha sido el aumento de la población inmigrante la que ha hecho que aparezcan nuevas aulas y necesidades. A pesar de todos estos cambios de población, en general el número de alumnos se ha mantenido constante desde su agrupación.

\section{Órganos de dirección y coordinación del centro}

Equipo Directivo

El Equipo Directivo aprovecha las horas de exclusiva para reunirse ya que por sus tareas de docencia es muy complicado que coincidan en un mismo centro.

Está formado por:

- La Directora, que además es tutora de una de las clases de $6^{\circ}$ de Primaria.

- La Secretaria, que además imparte clase de inglés.

- La Jefa de estudios, que itinera por los otros dos centros que forman el CRA, además de controlar la organización de los otros dos centros.

Una diferencia significativa con un colegio ordinario, es que en este CRA, es el Equipo Directivo quien tiene que hacerse cargo de la organización y funcionamiento del comedor.

\section{- $\quad$ La Dirección de un CRA}

Tiene su horario de docencia o de dirección dependiendo de las aules que tenga ese CRA. En concreto en este colegio, el horario de docencia es de 11 horas y el de dirección, de 14 horas, aunque el horario de dirección puede variar según las horas lectivas que se les otorguen. El Equipo Directivo tiene además la obligación de impartir clase a los cursos de niveles más altos, ya que estos ofrecen mayor nivel de independencia, de tal manera que los integrantes del Equipo Directivo puedan destinar el resto de su horario a tareas de dirección. 
Además la Directora tiene contacto permanente con los Ayuntamientos de los tres pueblos. Las dotaciones de recursos para estos centros, no sólo tienen su origen en la Administración Educativa (que da una sola dotación para todo el CRA, cuando en realidad, hay algunos materiales que se tienen que comprar por triplicado, dado que el CRA dispone de tres centros), sino que también son una gran fuente de ayuda los Ayuntamientos. De hecho, podrá apreciarse la mayor o menor implicación del Ayuntamiento de cada pueblo en sus propios centros.

\section{- La jefatura de estudios y Secretaría de un CRA}

Normalmente suelen estar destinados en otras localidades del CRA siendo frecuente el que sean tutores de grupos. Sus horarios se dividen, como en el resto de los centros en horas de docencia y horas para el ejercicio del cargo directivo, siendo esta asignación variable en función del número de aulas del mismo.

Las reuniones del equipo directivo se suelen hacer de forma semanal en la cabecera del CRA y sus funciones específicas, por la custodia de expedientes y organización general suele hacerse también en la cabecera, por lo que deben desplazarse en su caso varias veces a lo largo de la semana.

\section{Consejo Escolar}

Su constitución es diferente a la un Colegio Completo ya que está constituido por:

$\checkmark$ Un representante del Ayuntamiento de cada pueblo.

$\checkmark$ Padres de alumnos de los diferentes pueblos.

$\checkmark$ La Directora.

$\checkmark$ La Jefa de estudios.

$\checkmark$ Varios profesores que imparten clase en los diferentes centros.

$\checkmark$ La Secretaria.

$\checkmark$ Un representante de los alumnos,

Se reúnen en la cabecera mínimo una vez por trimestre, aunque en realidad intentan coincidir en más ocasiones. Sus funciones son las mismas que en cualquier centro educativo con la diferencia de la representatividad de todas las localidades. 
Se reúnen dos horas a la semana, en este CRA todos los miércoles por la tarde, ya que es el único momento en el que pueden coincidir todos. Para ello es necesario hacer ajustes en la organización horaria. El objetivo principal es trabajar en común y estar coordinados entre todos los profesores, tratan temas como las actividades que van a llevar a cabo como por ejemplo ahora están trabajando en la modificación de algún documento o la planificación del cambio de etapa en los niños de 5 años que van a pasar a primero. Se hace una planificación del trabajo, y en el programa Delphos que incluye una opción, se anexan todos los puntos que se tratan esa tarde. Este es otro de los métodos que se utiliza para mantener en contacto a los integrantes del CRA.

Tras esta reunión se juntan solo los coordinadores de ciclo para tratar de las programaciones del ciclo y de las necesidades del mismo, tienen un libro de actas que es una especie de diario donde se apunta el orden del día y se firma por todos los componentes, estos acuerdos serán comunicados al resto del profesorado a través de este acta. Además este libro de actas servirá para hacer la Memoria Anual.

Una diferencia esencial entre un colegio urbano y un CRA es que en el tema de reuniones, es necesario en este último establecer un día concreto y de obligatoria asistencia para estar coordinados, ya que además de la necesidad de juntarse todos hay que prever los desplazamientos a la cabecera. La queja más común por parte de sus componentes es que no se dispone del tiempo suficiente para tratar todos los temas de importancia.

Todos los asuntos de interés acordados se llevan la Comisión de Coordinación Pedagógica (CCP) formada por un coordinador de cada ciclo, incluido infantil, la directora, el orientador y el jefe de estudios, en ellos se toman acuerdos generales que han de servir para la organización pedagógica coordinada de todo el colegio.

\section{La orientación}

Hasta hace 4 años la orientadora era compartida con otros CRAs de la zona, ya que existía un equipo en Pastrana que los atendía, con lo que puede deducirse la falta de atención en el servicio, una queja muy común. Actualmente existe un orientador para este CRA. Éste, ofrece su apoyo por las distintas aulas, coordinándose además con el profesorado una vez por semana. Estudian y diagnostican casos de alumnos que son detectados por los tutores y sobre todo hacen tareas de asesoramiento y apoyo al profesorado en medidas de personalización de la educación y de atención a la diversidad. Cabe destacar que además del alumnado con déficits en mayor o menor grado y número, existe un aumento notable de población 
inmigrante a estas zonas rurales que necesitan ser atendidos y cambios organizativos y metodológicos dentro de modelos inclusivos exigen de la confluencia de acciones junto con el profesorado de aula de la orientación y del profesorado especialista en Pedagogía Terapéutica.

La forma de actuar de este equipo de apoyo es reuniendo a un máximo de 2-3 niños por ciclo, dependiendo de sus necesidades en una misma clase, la sala Althia, muy solicitada para realizar todo tipo de actividades que les permitan desarrollar al máximo sus potencialidades dentro de sus propios Programas de Trabajo Individualizados. En este CRA en concreto se opta por esta organización de atender alumnos de forma individual en pequeños grupos, en otros la opción es atenderlos dentro de la propia clase.

\section{Organización del centro}

Los Horarios

Como en el resto de los centros los horarios son confeccionados por el equipo directivo a principio de curso. Las singularidades de un CRA radican en que los horarios de los profesores especialistas cuando son itinerantes tienen una reducción horaria que varia en función del numero de kilómetros semanales, lo que implica al final menor numero de horas totales para el centro.

La dificultad de cuadrar horarios con esta singularidad y la necesidad de hacer coincidir a todos una tarde a la semana para coordinación son las dos pautas más importantes, el resto se hacen según las condiciones generales de horarios para las diferentes etapas educativas. A veces se necesitan agrupar horas de especialidades en un mismo día para evitar desplazamientos innecesarios. Con frecuencia se recurre a la estrategia de asignar como tutores de aulas a especialistas con lo que se reducen los desplazamientos a esas localidades.

En nuestro caso la mayoría de los profesores tutores de los centros de Illana y Almonacid son también itinerantes y deben aprovechar las horas de los recreos para desplazarse de un pueblo a otro, por lo que es complicado cuadrar todos los horarios de los profesores en los tres colegios.

Los recursos

El centro cuenta con los recursos individualizados de cada una de sus aulas para el alumnado, con aulas perfectamente equipadas, y al menos una sala para tutoría y/o reuniones en todas las localidades con un uso polivalente, bibliotecas de aula, materiales para 
especialidades de Música y Educación Física, ordenadores para el alumnado ${ }^{6}$ y los tutores. Las aulas de Almonacid y Albalate disponen de comedor escolar y todas ellas disponen de buenas instalaciones deportivas dentro de sus propias instalaciones.

Hay una falta de espacios importantes y generalizada en los CRA, concretamente otro aspecto significativo es el uso del Aula Althia cuyas funciones en este Colegio son como sala de ordenadores, clase de religión, clase de ingles, clase de música, biblioteca y desdobles, por lo que la jefa de estudios debe también crear un horario para esa sala debido a la gran diversidad de actividades que se realizan dentro de ella. Una peculiaridad es que en esta sala, las materias que requieren un profesor especialista, se reúnen a todos los alumnos de un mismo ciclo.

La cabecera del CRA cuenta con recursos centralizados como materiales especializados o recursos singulares, biblioteca general y del profesorado y toda la dotación de dirección y secretaría con conexión a internet. La asignación de recursos a cada localidad/aula se hace a través de las reuniones de coordinación de ciclos y se dispone de materiales fungibles en el centro cabecera que son retirados según necesidades. No obstante las dificultades con el tema de dotaciones es continua. Por ejemplo en el caso de este CRA, el dinero que reciben de la Administración se tiene que distribuir en tres partes, siendo el total más o menos el mismo que se le otorga a un colegio ordinario. Una de las consecuencias de este problema, es que en Almonacid no se disponga de ordenadores; en Illana, tras sufrir un robo, no se han podido reponer y en Albalate la red es a menudo deficiente.

Para recursos más específicos cuentan con el apoyo del Centro de Profesores de Guadalajara, del que dependen, del CTROADI ${ }^{7}$ para materiales de diferentes especialidades y materias y con Centro de Recursos para la Atención a la Diversidad con sede en el Colegio de Educación Especial Virgen del Amparo de Guadalajara.

Los documentos programáticos

\section{- Proyecto Educativo de Centro.}

El PEC de un CRA debe mantener toda la información que aportan los centros que forman el CRA. En general la línea y los puntos a seguir del PEC de un CRA son similares al de una escuela urbana. Los objetivos a plantear son algo diferentes dado que las necesidades

\footnotetext{
${ }^{6}$ Tras unos robos ocurridos hace algún tiempo todavía no se ha repuesto este material en algunas aulas.

${ }^{7}$ Los CTROADI, Centros de Recursos para la Orientación, Atención a la Diversidad e Interculturalidad se van a cerrar en el curso 09-10 pasando sus recursos a los CEP y Centros de Recursos para la Atención a la Diversidad.
} 
también lo son, como puede ser por ejemplo, la dificultad para llegar a los colegios, las agrupaciones de alumnos de distintos niveles en una misma aula y en consecuencia la organización de las sesiones y los horarios del personal.

A rasgos generales, los puntos que este CRA incluye en este documento son:

- Las señas de identidad y situación de los tres centros.

- Las características del profesorado que compone el claustro que, en general tienen muy pocos profesores definitivos.

- Características de las familias de los alumnos.

- Principios educativos y valores que guían los tres centros, contenidos en la LOE, pero escogidos aquellos que más se adecuen a los requisitos del CRA.

- Etapas del centro y objetivos generales de cada ciclo.

- La colaboración que mantienen con otras instituciones como el Ayuntamiento, la administración, el AMPA, los servicios sociales...

- Las jornadas del centro y los horarios e itinerancias del profesorado

- La ruta del transporte escolar, el cual pasa por varios pueblos, entre ellos Sayatón y Bolarques, dependientes del Ayuntamiento de Almonacid. En este punto hay que destacar que acuden a la cabecera del CRA a pesar de que el colegio de Almonacid lo tienen más cercano.

- $\quad$ La organización del comedor, en este caso, dirigido por el Equipo Directivo.

- El plan de evaluación interna, que cambia cada año dependiendo de los puntos débiles de cada centro dentro de cada curso escolar (obras, problemas de convivencia,...)

- Si anteriormente se incluía el Proyecto Curricular de Centro, ahora se incluyen las Programaciones Didácticas donde se trata la atención al alumnado, la diversidad de grupos, su distribución,...como por ejemplo, este año, en el Colegio Santa Cruz (la cabecera), por falta de alumnado han tenido que juntar por un lado a los alumnos de segundo ciclo en un mismo aula y por otro a los de tercer ciclo.

- El Plan de Atención y Convivencia, elaborado por el equipo de orientación y apoyo, con ayuda del Equipo Directivo.

Como ocurre habitualmente, las modificaciones que se hacen sobre el PEC deben quedar plasmadas en la Memoria Anual, pero algo significativo en los Colegios Rurales es que estos cambios son muy constantes. Por ejemplo, si aumentan los alumnos en los 
diferentes centros y se convierten en independientes, entonces el PEC deberá cambiar completamente dado que las necesidades y la nueva situación del centro son diferentes.

\section{- Programación General Anual}

Elaborada por el Equipo Directivo al principio del curso. Deben realizar consultas en los otros dos centros que componen el CRA para que los objetivos que incluyan estén acordes con las necesidades de los tres centros. El Equipo Directivo recoge las conclusiones y los problemas detectados el curso anterior y los transforman en objetivos para el curso siguiente. Este documento contiene algunas partes que necesitan de la ayuda de la Orientación.

Contiene los horarios de los profesores y de los alumnos, la planificación en las sustituciones, las actividades extraescolares (escasas), presupuesto de gastos (realizado por el secretario, basándose en el gasto del curso pasado), la Evaluación Interna del Centro, calendario de reuniones de especialistas, apoyos, etc.

Como anexo, y aquí radica la gran diferencia con respecto a Programaciones de centros ordinarios, es que recogen los planes de trabajo de cada uno de los centros, y especialmente se centra en los requisitos mínimos para una buena preparación de los alumnos que terminan sexto de Primaria, hacia Secundaria.

La atención a la diversidad

¿Cómo se atiende en la programación didáctica la diversidad de alumnos que componen estos centros? La principal actuación que llevan a cabo es la coordinación entre los profesores, fundamentalmente posible gracias a las reuniones semanales y el aprovechamiento de las horas complementarias.

Al profesorado de este CRA le interesa responder a la diversidad del alumnado y de situaciones, así toma decisiones y diseña su práctica dentro de un currículum abierto y flexible. Por ello, el profesorado deberá informarse de sus alumnos revisando el expediente de cursos pasados. La directora comenta que antes de elaborar un Plan de Trabajo Individualizado (PTI) se deja un mes para tratar con el aula con la que van a trabajar a lo largo del curso. Este PTI lo elaboran todos los profesores y posteriormente se incluye en la Programación Didáctica.

Se utiliza también como recurso el hecho de tener alumnos de diferentes edades en una misma clase, ya que entre los mismos alumnos se producirá el proceso de Enseñanza Aprendizaje. Con esto queremos decir que hay más oportunidades de aprendizaje para los 
niños; los de cursos inferiores aprenden cuando escuchan las clases que se dan para los alumnos de cursos superiores, y estos últimos también aprenden más cuando escuchan las clases que se imparten a los de menor edad, es decir, es como un avance para los primeros, y como un repaso para los últimos.

Los profesores además intentan adecuar los contenidos del temario dependiendo de la agrupación que tenga su clase, de forma que todos los alumnos puedan entenderlo e interiorizarlo, asegurándose de que todos, tengan diferentes edades o no, lo hayan aprendido.

Muchas veces es difícil atender a las exigencias de la Administración Educativa debido a la limitación de recursos, es decir, por un lado te imponen trabajar con las Nuevas Tecnologías, pero existe el problema de que en muchos sitios la red no llega correctamente y es imposible que los alumnos alcancen los objetivos que se exigen.

\section{La gestión de la convivencia}

En general, las normas de convivencia de un CRA son las mismas que para un colegio urbano, deben estar contenidas en el Plan de Convivencia, y deben recoger, además, todas las medidas correctivas o sanciones y las formas de gestionar la convivencia que el centro quiera adoptar y que deben aplicar dependiendo de la norma incumplida por un alumnado.

Las normas de convivencia son iguales para todos los centros, pero adaptadas a las características del medio rural, y son extraídas igualmente de la LOE. En estas se incluyen los derechos y deberes tanto de las familias, como de los alumnos y del profesorado. En este caso en concreto las normas generales van a ser propuestas por el Claustro de Profesores, consensuadas con padres y aprobados en Consejo Escolar y no se modifican a menos que se de una circunstancia especial o que cambie la normativa. Las normas más específicas, las de aula, las elabora el tutor con su grupo de alumnos, y son diferentes para cada clase.

La forma de actuar de este centro es la siguiente; si tras hablar con el alumno, éste continua con el mismo comportamiento, el profesor tutor lo comenta con los padres para que estos tengan constancia de tal comportamiento, si tras un cierto tiempo, el alumno continua con el mismo comportamiento, la directora puede imponer una sanción y a la vez se pone en contacto con Servicios Sociales, los cuales llevarán a cabo las medidas oportunas. Si los padres no estuviesen de acuerdo con estas correcciones, es el Consejo Escolar el que se hace cargo, se reúne y toman una decisión. 
Según nos comenta la directora. En el próximo curso habrá una Comisión de Absentismo, que servirá para tomar medidas cuando los alumnos faltan al colegio. En esta Comisión, se crearán nuevas normas, actividades más motivadoras..., habrá una representación del Ayuntamiento, de los padres y de Servicios Sociales.

Los proyectos singulares

Con este apartado nos queremos referir al menos a dos actuaciones que en forma de proyectos desarrollan este tipo de centros y que son muy características.

\section{- Las convivencias o semanas culturales.}

La mayoría de los CRAs realizan a lo largo del curso una o varias jornadas de convivencia, ya sean en el marco de semanas culturales o no. Consisten en juntar a todos los alumnos del CRA en una misma localidad para desarrollar una serie de tareas programadas con el doble objetivo de implementar el currículo y de establecer relaciones más estrechas entre el alumnado de las diferentes localidades de forma que se conozcan, convivan y desarrollen tareas conjuntamente.

El CRA la Colmena ha llevado a cabo este año estas jornadas en la localidad de .... Y han estado ejecutadas en torno al tema de....

- Los proyectos de innovación.

Cada vez con más frecuencia estos centros suelen presentarse a convocatorias de Proyectos de Innovación o de Proyectos de Aprendizaje Permanente de forma que se establecen entre todos los colegios una relación más estrecha de un trabajo conjunto con un mismo fin.

El desarrollo de estos proyectos es muy importante para los CRAs pues puede desarrollar acciones muy propias de la zona rural en la que se desarrollan, concretamente algún CRA esta trabajando en temas relacionados con medio ambiente y ecología.

El CRA La Colmena no esta desarrollando en este momento ningún Proyecto de este tipo, pero ha estado desarrollando este curso un Grupo de Trabajo de creación de materiales para el desarrollo de las competencias en el alumnado.

\section{¿De qué forma dan apoyo los CRAER a UN CRA?}


Los Centros de Recursos y Asesoramiento a la Escuela Rural tienen el objetivo de coordinar, asesorar y formar a los profesores de los centros de su entorno, así mismo deberían mantener contacto asiduamente, en cambio. La realidad es diferente debido a la lejanía de estos centros, lo que suele ocurrir es que la relación más continua es con la cabecera y el equipo directivo y con el resto de forma telefónica, y en función de las necesidades planteadas se estudia en equipo la mejor y más adaptada respuesta.

Los CRAER dan un apoyo singular a los CRA a través de la asesoría de Orientación, para el diagnóstico del alumnado y la realización de medidas específicas, siendo continua la presencia de estos en los CRA y un referente aunque no formen parte de su plantilla orgánica. Por otro lado la proximidad de estos sirve a veces como complementación y facilitador de recursos para actividades varias: creación de revistas, edición de videos, publicación de materiales...

En los casos en los que no existen CRAER, los Centros de Profesores son los encargados de ofrecer un contacto más directo con los CRA, esto se lleva a cabo mediante el asesoramiento directo y la formación del profesorado. Muchas veces los centros recurren a grupos de trabajo o seminarios en sus propios centros para dar respuesta a sus necesidades formativas o de generación de materiales o documentos. Los temas tratados en estos grupos de trabajo, son recogidos en actas por los profesores y posteriormente son incluidos en la Memoria Anual, además realizan también una memoria de grupo que es enviada a los CEP.

Para mejorar la respuesta una de las propuestas es convocar en los CRAER al final del curso y una vez por año las jornadas de intercambio de experiencias, donde se reúnen todos los profesores de los centros de la provincia y crean debates exponiendo los resultados positivos o negativos y las experiencias que han vivido durante ese curso escolar, es una forma de ayudar a los demás profesores para que conozcan cómo podrían resolver un problema, qué hacer, a quién acudir, para resolverlo con la mayor rapidez y eficacia posible.

En concreto en este CRA, tiene como referencia el CEP de Guadalajara, y tienen un asesor asignado por cada perfil para que pueda resolverles los problemas que puedan surgir o asesorarles en el desarrollo de tareas. Desde el CRA cuando se necesita el profesorado asiste a cursos de formación específicos según sus necesidades.

Anteriormente existía un centro de profesores en Pastrana que permitía tanto a los CRA como a los asesores mantener una estrecha relación y recibir visitas más continuas. Actualmente este contacto es más de tipo telefónico o presencial en situaciones concretas.

\section{¿Qué relación mantienen con los CRIE?}


Dichos centros complementan y refuerzan el papel de los Colegios Rurales Agrupados de una misma provincia. Normalmente el CRIE de una determinada zona mantiene contacto directo y permanente con los CRA de su zona y se programan las actividades a realizar y los calendarios para las actuaciones.

En las visitas a los CRIE el profesorado de los CRA juega un papel muy importante ya que de un lado se van a desarrollar sus programaciones desde una perspectiva más lúdica y atractiva para el alumnado y de otro porque le permite a través de la convivencia diaria con sus alumnos valorar y evaluar otras competencias.

El problema reside en que son poco numerosos y en concreto en la provincia de Guadalajara no se dispone de ningún CRIE, siendo únicamente el más cercano el CRIE de Cuenca con el que este CRA no mantiene ninguna relación. Además, según nos comentan es muy complicado que acepten la solicitud para que los alumnos participen en las actividades que ofrece este CRIE.

\section{Conclusiones}

Las diferencias y peculiaridades territoriales han configurado modelos educativos desiguales con necesidades y soluciones diferenciadas para la escuela rural. Según las regiones la estabilidad de los equipos es mayor e incentivada; los complementos y reconocimiento de la escuela rural son diferentes; los niños pueden llevar o no acompañante en el transporte escolar; los libros pueden ser gratis; la compensación económica por kilometraje de los profesores itinerantes es diferente y un sinfín de diferencias; aunque las quejas parece que van todas en la misma dirección: un alto porcentaje de profesorado interino que baila de un pueblo a otro hasta conseguir su plaza en la ciudad y que crea inestabilidad en el alumnado; poco dinero para gastos de funcionamiento y un presupuesto escaso que se reparte con igual criterio en la ciudad y en el campo, cuando sus necesidades no son las mismas; el nulo reconocimiento a una labor ardua y difícil y en un destacado lugar la falta de preparación para la docencia en las condiciones de la escuela rural.

Se asume como primer requisito que los profesores tienen que conocer mejor la cultura y el medio social de los alumnos para poder construir su propuesta pedagógica. Sin embargo, existe un choque cultural del docente que no conoce el medio rural ni su escuela por experiencia propia, lo que constituye un obstáculo, pues es una herramienta primordial la construcción de todo el proceso educativo sobre la base de los códigos y las experiencias que ya posee el niño. Este es un aspecto que todos los profesores con los que hemos tratado han 
comentado; no existe una formación específica acerca del trabajo en escuelas rurales, por lo que ha de improvisarse todo sobre la marcha.

Por lo general, cuando el profesorado llega a una escuela rural lo hace por falta de experiencia, aunque mínimamente la administración intenta potenciar la llegada y permanencia de profesorado en estos centros a través de incentivos

El reciente estudio de Barley y Beesley (2007) pone de manifiesto la relevancia de la retención de maestros para lograr el apoyo de la comunidad y aumentar las tasas de rendimiento académico de su alumnado.

Por otro lado la existencia de buenas vías de comunicación y el acceso a los medios particulares de transporte hace con que este profesorado se desplace a diario hasta las grandes ciudades para vivir, con la consiguiente pérdida del conocimiento de la realidad en la viven sus alumnos y las condiciones y recursos del entorno próximo. Las conexiones personales de los profesores con el medio contribuyen al éxito de estas escuelas, permitiendo el compromiso y la dedicación. Sin embargo, las familias perciben de manera habitual que el profesorado encuentra en la escuela del pueblo un lugar de paso, lo que dificulta el desarrollo de proyectos escolares y locales a largo plazo, siendo esta sea una queja permanente de los padres.

Estas ventajas e inconvenientes forman parte del día a día de la escuela rural, constituyendo un mosaico de piezas que conforman una realidad tan atractiva como desdeñable y cuyo buen funcionamiento depende, en la mayoría de casos, de la buena disposición del maestro. Así, no siempre las circunstancias tienen por qué ser o vivirse desfavorablemente. El maestro que inicia su carrera docente en tales centros encuentra elementos positivos que pueden hacer que su tarea se vea valorada, convirtiéndose en una pieza clave del entramado rural. En algunos casos, las ganas de trabajar de este profesorado novel producen avances y experiencias educativas que pueden ser compensadoras de los déficits formativos.

Por otro lado y como hemos visto:

- Los centros tienen buenas dotaciones de infraestructura y de recursos, aunque suelen faltar dependencias dentro de los centros para atender a todas las actividades que en ellos se desarrollan.

- Hay una queja generalizada de falta de espacios en este tipo de centros.

- Los órganos de dirección tienen exceso de funciones y tareas a desarrollar, al acumular docencia y dirección de varios centros.

- Los equipos directivos se quejan de la falta de recursos para desarrollar los objetivos y proyectos. 
- Existe en general una alta rotatividad del profesorado de los centros rurales lo que dificulta el desarrollo de proyectos a largo plazo.

- La existencia de profesorado itinerante conlleva dos dificultades, de un lado de organización de horarios y de otro el relacionado con la compensación de horas.

- El profesorado tiene que desplazarse con asiduidad para desarrollar sus tareas, lo que genera problemas de coordinación y de cumplimiento de objetivos.

- El número de profesores demasiado ajustado genera problemas por la necesidad de cumplir en primer lugar las sustituciones de faltas.

- No hay un criterio diferenciador de objetivos en los documentos organizativos por el hecho de ser un colegio rural.

- Existen diferencias en los aspectos organizativos y de coordinación en los documentos organizativos.

- Se desarrolla como proyecto general de los CRA las jornadas de convivencia del alumnado.

\section{A STUDY ABOUT THE SPANISH COUNTRY SCHOOL}

ABSTRACT: In this study research we try to study the elements that involved the rural school, from the concept and the organization, to the work of the management team, teachers that travel from one village to another, how to work with the diversity of students in the same classroom...urthermore, this research offers us a description of the situation at rural schools; this work has been achieved through observation and collect of oral information about the reality of teachers, students, regulation and structure in a rural school. For it, we have gone to a rural school, placed in a village of Guadalajara, named Grouped Rural School "La Colmena", who is the head of three schools of the province of placed in Albalate of Zorita, Almonacid de Zorita and Illana.

KEYWORDS: School Organization, Grouped Rural School, Resources and Advice Center for Rural School, Rural centers of educational innovation, unitary schools/unitary classrooms, itinerant teachers.

\section{REFERENCIAS}

BARLEY, Z.A.; BEESLEY, A. D. Rural school success: what can we learn? Journal of Research in Rural Education, Rackley Building, v.22, n.1, p.1-16, jan. 2007.

BUSTOS JIMÉNEZ, A. Los grupos multigrado de educación primaria en Andalucía. 2006. 512f. Tesis (Doctoral en Didáctica y Organización Escolar) - Universidad de Granda, Granada, 2006. 
BOIX TOMÁS, R. Estrategias y recursos didácticos en la escuela rural. Barcelona: Graó, 1995.

FEU, J. La escuela rural en España: apuntes para un debate. Cuadernos de Pedagogía, Barcelona, v. 327, p.90-94, sept. 2003.

ESPAÑA. MINISTERIO DE EDUCACIÓN [MEC]. Informe sobre el estado y situación del sistema educativo (2004-2005). 2007. Disponible en:

<http://www.mec.es/cesces/informe-2004-2005/b-indice.pdf>. Acceso en: 19 sept. 2008.

PLIYA, J. Tendencias recientes de la educación en los países en desarrollo y, especialmente, en África sudsahariana. In: UNESCO. Sobre el futuro de la educación: hacia el año 2000. Madrid: Narcea, 1990.

ROCHE ARNAS, P. Los centros rurales de innovación educativa de Teruel (CRIET): un intento de solución al problema de la Escuela Rural. Zaragoza: ICE de la Universidad de Zaragoza, 1992.

\section{WEBS CONSULTADAS}

CONSEJO ESCOLAR. Informe 1/2009 del Consejo Escolar sobre el inicio del curso 200809 en los Centros Públicos de Castilla-la Mancha. Disponible en:

$<$ www.educa.jccm.es/educa-

$\mathrm{jccm} / \mathrm{cm} /$ educa_jccm/images?locale=es_ES\&textOnly=false\&idMmedia=86635> . Acceso en: 19 sept. 2008.

Informe 2/2007 del Consejo Escolar sobre el inicio del curso 2007-08 en los centros públicos de Castilla-la Mancha. Disponible en:

$<$ http://www.educa.jccm.es/educa-

$\mathrm{jccm} / \mathrm{cm} /$ educa_jccm/images?locale=es_ES\&textOnly=false\&idMmedia=40944>. Acceso en: 19 sept. 2008.

GUÍA DEL ESTUDIANTE. Oferta educativa. Disponible en:

$<$ http://www.apepalen.cyl.com/guia_estudiante/guia0910/9.pdf>. Acceso en: 19 sept. 2008.

OBJETIVOS del Programa Aldea Digital. Disponible en:

$<$ http://centros4.pntic.mec.es/cp.ribera.del.duero/descripcion21.htm>. Acceso en: 19 sept. 2008.

PORTAL DE EDUCACIÓN. Información e servicios para: Profesorado. Disponible en: <http://www.educa.jccm.es/educa-jccm/cm/temas/19700011>. Acceso en: 19 sept. 2008.

COLÉGIO Agrupado Rural. In: WIKIPEDIA a enciclopédia libre. Disponible en: $<$ http://es.wikipedia.org/wiki/Colegio_rural_agrupado>. Acceso en: 19 sept. 2008. 